\title{
Plasma lens-based beam extraction and removal system for plasma wakefield acceleration experiments
}

\author{
R. Pompili®, ${ }^{1,{ }^{*}}$ E. Chiadroni, ${ }^{1}$ A. Cianchi, ${ }^{2}$ A. Del Dotto, ${ }^{1}$ L. Faillace, ${ }^{3}$ \\ M. Ferrario, ${ }^{1}$ P. Iovine, ${ }^{4}$ and M. R. Masullo ${ }^{4}$ \\ ${ }^{1}$ Laboratori Nazionali di Frascati, Via Enrico Fermi 40, 00044 Frascati, Italy \\ ${ }^{2}$ INFN and Tor Vergata University, Via Ricerca Scientifica 1, 00133 Rome, Italy \\ ${ }^{3}$ INFN Milano, via Celoria 16, 20133 Milan, Italy \\ ${ }^{4}$ INFN Napoli, Via Cintia, 80126 Naples, Italy
}

(Received 6 September 2019; published 30 December 2019)

\begin{abstract}
Plasma wakefield acceleration represents one of the most promising techniques able to overcome the limits of conventional $\mathrm{rf}$ technology and make possible the development of compact accelerators. With respect to laser-driven schemes, the particle beam-driven scenario is not limited by diffraction and dephasing issues; thus, it allows one to achieve larger acceleration lengths. Nevertheless, one of the most prominent drawbacks occurs at the end of the acceleration process and consists in the removal of the depleted high-charge driver while preserving the main features (emittance and peak current) of the accelerated witness bunch. Here we present a theoretical study demonstrating the possibility to reach these goals by using an innovative system consisting of an array of beam collimators and discharge capillaries operating as active-plasma lenses. Such a system allows one to extract and transport the accelerated and highly divergent witness bunch and, at the same time, provides for the removal of the driver. The study is completed with a set of numerical simulations conducted for different beam configurations. The physics of the interaction of particles with collimator is also investigated.
\end{abstract}

DOI: 10.1103/PhysRevAccelBeams.22.121302

\section{INTRODUCTION}

Plasma-based acceleration, driven by either ultrashort laser pulses [1-3] or electron bunches [4-6], represents one of the most promising techniques able to overcome the limits of conventional rf technology and allow the development of compact accelerators. In both schemes the plasma is used as an energy transformer in which the driver pulse energy is transferred to the plasma (through the excitation of plasma wakes) and, in turn, to a witness bunch (externally [7,8] or self-injected $[9,10])$. Plasma wakefield acceleration (PWFA) driven by one or more driver bunches offers several advantages with respect to laser-driven schemes, mainly limited in their overall efficiency and in the maximum accelerating lengths that can be achieved considering the laser diffraction, dephasing, and depletion [11]. At the FACET facility at SLAC laboratories [6], for instance, it has been experimentally demonstrated that by employing driver beams with $\mathrm{GeV}$ energies the overall efficiency can be boosted up to $30 \%$ on a $40-\mathrm{cm}$-long plasma stage. It

\footnotetext{
*riccardo.pompili@lnf.infn.it
}

Published by the American Physical Society under the terms of the Creative Commons Attribution 4.0 International license. Further distribution of this work must maintain attribution to the author(s) and the published article's title, journal citation, and DOI. represents the starting point for future facilities based on plasma acceleration like, for instance, the European Plasma Research Accelerator with excellence in applications (EuPRAXIA) [12].

A drawback common to both the laser and particle beamdriven methods is represented by the extraction of the accelerated witness bunch that can lead to a large degradation of its emittance [13]. When exiting the plasma, the bunch has a large angular divergence [14], of several milliradians, that is some orders of magnitude larger with respect to conventional (rf) accelerator beams. For non-negligible energy spreads, such a large divergence also leads to a rapid increase of the normalized emittance in the downstream drift [15]. It is thus mandatory to remove such a divergence as soon as the witness leaves the plasma accelerator. In addition to this, for PWFA another issue is represented by the removal of the high-charge energy-depleted driver(s) [16]. In this case, it is evident that simple collimating apertures cannot be employed without affecting also the witness charge.

With the aim to solve these issues, here we discuss an innovative and compact extraction system that implements an array of active-plasma lenses [17-19] consisting of multiple discharge-waveguide capillaries filled with gas. Taking advantage of the energy-dependent focusing ( $\propto \gamma^{-1}$, with $\gamma$ the relativistic Lorentz factor) provided by an activeplasma lens [20,21], such a solution allows one to refocus and transport the witness along the array while overfocusing 
the (lower-energy) driver that is removed by using a collimator located between the lenses. Our study indicates that the entire system can be made very compact (tens of centimeters scale) and adapted to different beam configurations.

The paper is organized as follows. Section II describes the reference scenario, i.e., a typical driver-witness beam configuration as the one foreseen for the proposed EuPRAXIA [12] design study. In Sec. III, we describe the conceptual design of the extraction system and discuss the working regime of the employed plasma lenses. Beam dynamics simulations are then reported in Sec. IV, where the scalability of the system is tested for several beam configurations. In Sec. V, we describe the interactions of the particle beams with the collimator device. The analysis highlights the effects of the wakefields generated in the collimator aperture (Sec. VA) and the particle-matter interactions by means of the GEANT4 framework (Sec. V B), allowing one to evaluate the effective removal of the driver particles and the effects of their interaction with the surrounding materials.

\section{THE EuPRAXIA DESIGN STUDY}

In the context of accelerator research, a fundamental milestone is represented by the realization of a plasmadriven facility that will integrate high-gradient accelerating plasma modules with a short-wavelength free electron laser (FEL). In such a context, the Horizon 2020 design study EuPRAXIA [22] aims to design the world's first accelerator facility based on plasma-wakefield acceleration to deliver 1-5 GeV electron beams that simultaneously have high charge and low normalized emittance and energy spread, as required by user applications [12]. The EuPRAXIA
Collaboration concluded their work by suggesting a solid strategy with the aim to demonstrate the possibility to use plasma accelerators in such a context. The design study proposes a first European research infrastructure that is dedicated to demonstrating exploitation of plasma accelerators for final users. Developing a consistent set of beam parameters produced by a plasma accelerator able to drive a short-wavelength FEL is one of the major commitments of the design study. To prove such a concept, EuPRAXIA foresees the realization of two facilities that will be the pillars of the design study. One will exploit the use of highpower lasers to generate the plasma wakes and provide the acceleration of an externally injected witness bunch [23]. The other one will use a beam-driven scheme $[4,6]$, where the plasma wakefields are generated by one or more preaccelerated electron bunches.

For the beam-driven scenario, the LNF-INFN laboratories located in Frascati have been recently proposed as possible hosting site [24,25], and a conceptual design report of the foreseen facility has been recently produced [26]. To generate FEL radiation with wavelengths in the socalled water-window range $\left(\lambda_{\mathrm{FEL}} \approx 2.5-4 \mathrm{~nm}\right)$, such a facility will employ an $X$-band linac to produce and preaccelerate a driver-witness beam up to $500 \mathrm{MeV}$ and then inject it into a PWFA-based booster. The beam-driven plasma acceleration module will operate in the so-called quasinonlinear regime [27] with plasma densities of the order of $n_{p} \approx 10^{16} \mathrm{~cm}^{-3}$ and will reach $1 \mathrm{GeV}$ final energy for the witness bunch [28,29]. Figure 1(a) shows the simulated longitudinal phase space (LPS) of a $200 \mathrm{pC}$ driver followed by a $30 \mathrm{pC}$ witness downstream from the PWFA module. The results are obtained using ARCHITECT [30], a hybrid code that works as a particle in cell for the electron bunches while treating the plasma as a fluid.
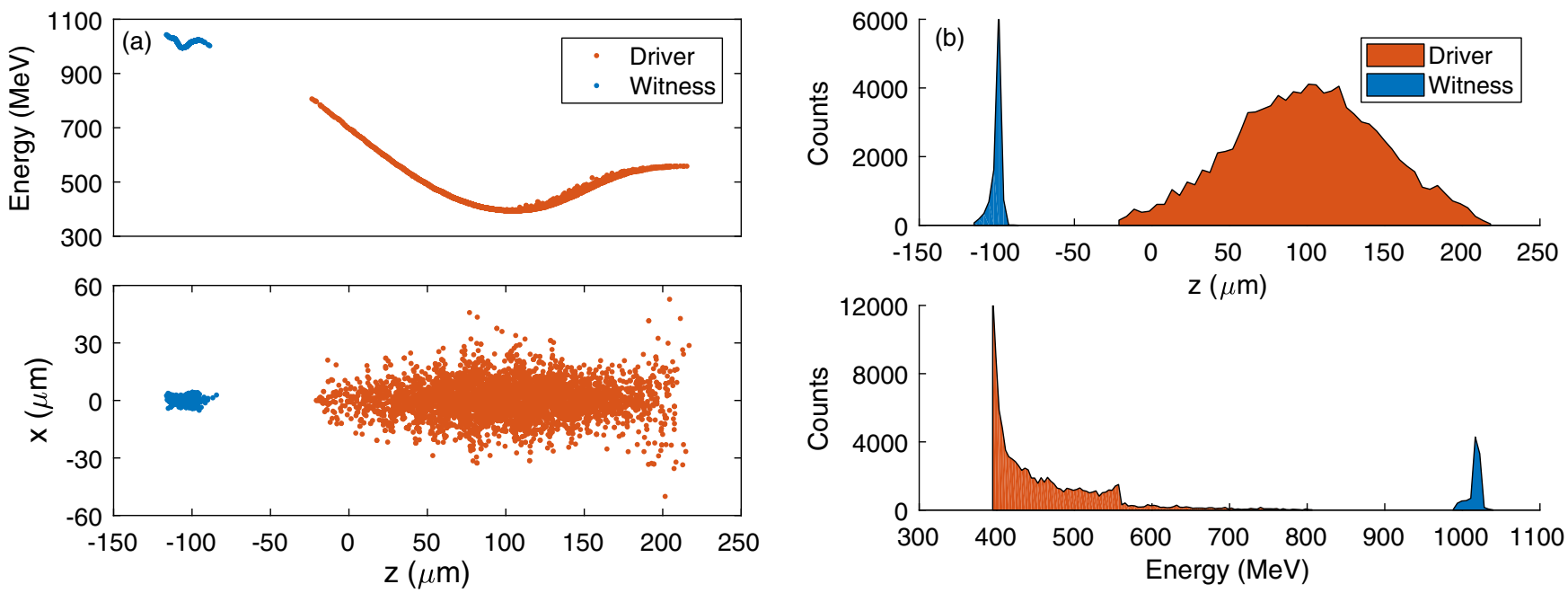

FIG. 1. (a) Longitudinal phase-space (top) and $x-z$ view (bottom) of the accelerated witness (blue) and energy-depleted driver (red) bunches downstream the PWFA module. Before the plasma acceleration stage, the energy of both bunches was $500 \mathrm{MeV}$. (b) Longitudinal (top) and energy (bottom) profiles of the two bunches. The $y$ axis reports the counts of the simulated macroparticles for each bunch. 
TABLE I. Witness and driver bunches parameters at the exit of the PWFA module.

\begin{tabular}{lccc}
\hline \hline Parameter & Units & Witness & Driver \\
\hline Charge & $\mathrm{pC}$ & 30 & 200 \\
Duration (rms) & $\mathrm{fs}$ & 11.5 & 160 \\
Peak current & $\mathrm{kA}$ & 2.6 & 1.2 \\
Energy & $\mathrm{MeV}$ & 1016 & 460 \\
Energy spread (rms) & $\%$ & 0.73 & 16 \\
Normalized emittance & $\mu \mathrm{m}$ & 0.6 & 5 \\
Spot size & $\mu \mathrm{m}$ & 1.2 & 7 \\
\hline \hline
\end{tabular}

The energy of the two bunches was $500 \mathrm{MeV}$ upstream from the PWFA stage, and, as shown in Fig. 1(b), most of the driver energy has been depleted into the plasma and converted in wakefields used to accelerate the witness bunch. The simulation has been performed to demonstrate that it is possible to preserve the witness bunch quality (normalized emittance and energy spread) during the acceleration in a plasma channel [16]. The main parameters of the two bunches at the exit of the PWFA module are reported in Table I.

\section{EXTRACTION SYSTEM}

When exiting the plasma (with density $n_{p}$ ), the witness moves from an extremely intense focusing field (generated by the excited plasma wakefield) to a free space, where the focusing effect suddenly vanishes. Assuming that the bunch was transversely matched into the plasma channel, its particles oscillate with a typical betatron $\beta$ function of amplitude $\beta_{e q}=\sqrt{\gamma / 2 \pi r_{e} n_{p}}$, with $r_{e}$ the classical electron radius [31,32]. At the plasma exit, the average divergence is thus given by $\sigma_{x^{\prime}} \propto 1 / \beta_{e q}$. As a reference case, when considering $n_{p} \approx 10^{16} \mathrm{~cm}^{-3}$ and $\gamma \approx 2000$ (i.e., $1 \mathrm{GeV}$ energy), one has $\beta_{e q} \approx 3 \mathrm{~mm}$. Considering typical normalized emittances of $\epsilon_{n} \approx 1 \mu \mathrm{m}$, the angular divergence is of several milliradians, i.e., orders of magnitude larger than the ones coming from conventional rf accelerators (of the order of microradians). For non-negligible energy spreads $\sigma_{E}$, such a large divergence also leads to a rapid increase of the normalized emittance in a drift of length $s$ given by [15]

$$
\epsilon_{n}^{2}=\langle\gamma\rangle^{2}\left(s^{2}\left(\frac{\sigma_{E}}{E}\right)^{2} \sigma_{x^{\prime}}^{4}+\epsilon_{g}^{2}\right),
$$

where $E$ and $\epsilon_{g}$ are the bunch energy and geometrical emittance, respectively. According to Eq. (1), it is thus important to remove the divergence of the accelerated witness bunch with a short focal length focusing system installed downstream from the plasma stage and as close as possible to it [33].

The technique we discuss here has two goals: (i) transport the witness bunch by preserving its charge, normalized emittance and peak current; (ii) remove the high-charge

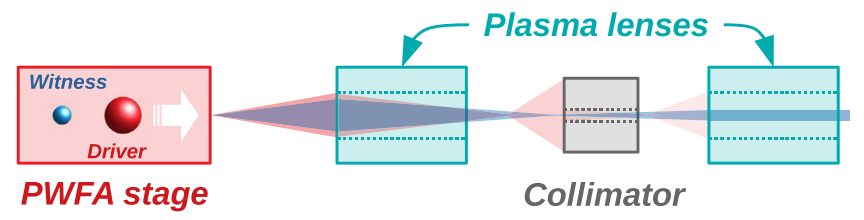

FIG. 2. Layout of the extraction system consisting of two active-plasma lenses employed as plasma lenses and a lead collimator between them. The focusing strengths of the lenses are set up to rapidly catch the witness bunch (blue) downstream from the PWFA stage and allow for its transport without any loss of charge. The driver bunch (red) has a lower average energy and is thus overfocused: Its spot size at the collimator entrance is larger than the aperture, and its charge is thus cut proportionally.

driver bunch during the transport in order to completely discard it before the FEL undulator beam line. Figure 2 shows the layout of such a system. The focusing of the witness is provided by two active-plasma lenses (APLs) [34], such devices being able to produce large focusing fields of the order of $\mathrm{kT} / \mathrm{m}$ [17]. Between the APLs, a lead collimator is used to remove the driver bunch. The removal is based on the different focusing provided by the first APL to the two beams: The witness is focused exactly at the entrance of the collimator (without losing any charge), while the driver (with approximately half of the energy) is overfocused to a spot size much larger than the collimator clear aperture.

To achieve the desired goals, i.e., focus the high-current and low-emittance witness bunch and dump the highcharge and energy-depleted driver, several simulation tools have been used and linked to each other. As described in Sec. II, the plasma acceleration process was simulated with the ARCHITECT code. To propagate and transport the two bunches from the exit of the PWFA module, we have used the general particle tracer (GPT) code [35], a tool widely employed in the accelerator community. The GPT has thus been used to simulate the drifts between all the elements involved in the system depicted in Fig. 2 with space-charge effects included. The dynamics of the beam in the plasma lenses is then computed by a $2 \mathrm{D}$ code written to solve the wakefield equations in the linear regime [36]. More detail are given in Sec. IVA. Finally, the optimization of the collimator system has been conducted to maximize the driver beam dumping by looking at its fundamental interactions with the collimator walls (predicted by GEANT4; see Sec. VB) and by analyzing the effects, on the witness beam, of the wakefields produced during the propagation through the collimator aperture (with the CST simulation framework, Sec. VA).

\section{A. Active-plasma lens}

The core part of the system is represented by the activeplasma lenses. An APL essentially consists of a currentcarrying cylindrical conductor whose axis is parallel to the 
beam. Here the plasma (produced after the ionization of the gas confined within the capillary) acts only as a conductor, while the net focusing effect is produced by the flowing discharge current. A schematic picture of such a device is shown in Fig. 3. By indicating with $J(r)$ the current density along the radial dimension, the resulting magnetic field is given by the Ampere law

$$
B_{\mathrm{ext}}(r)=\frac{\mu_{0}}{r} \int_{0}^{r} J\left(r^{\prime}\right) r^{\prime} d r^{\prime},
$$

where $\mu_{0}$ is the vacuum permeability. If the current density is uniform, the magnetic field increases linearly with the radius $r$, and a linear restoring force on the beam will result. One can notice three interesting features of such a device. (i) The focusing is symmetric, like in solenoids, but the resulting force scales as $F \propto \gamma^{-1}$ (with $\gamma$ the relativistic Lorentz factor), like in quadrupoles. (ii) The focusing can reach several tens of $\mathrm{kT} / \mathrm{m}$, i.e., orders of magnitude larger than the strongest available quadrupoles $(\approx 600 \mathrm{~T} / \mathrm{m}$ $[37,38])$. (iii) The tunability of the system is obtained by adjusting the external discharge current $I_{D}=\int_{S} \mathbf{J} \cdot d \mathbf{S}$.

These features pushed the research toward the use of APLs in accelerator facilities. While in past decades several results were obtained by focusing ion beams [39-41], recently several proof-of-principle experiments have been performed with laser-plasma $[17,20]$ and rf $[18,42-44]$ accelerators. Although these results indicated the capability to integrate APLs in accelerator facilities and their advantages (in terms of focusing gradients) with respect to conventional optics, some of them have demonstrated that nonuniformities on the $J(r)$ and the interaction of the traveling beam with the background plasma can induce severe effects on the beam itself, in particular, on its emittance [18,42].

The focusing field produced by the APL strongly depends on the discharge dynamics along the capillary $[45,46]$. To describe the main effects of the discharge process, a one-dimensional analytical model can be used [47] by assuming the distribution of plasma inside the capillary at the equilibrium stage as soon as the discharge is

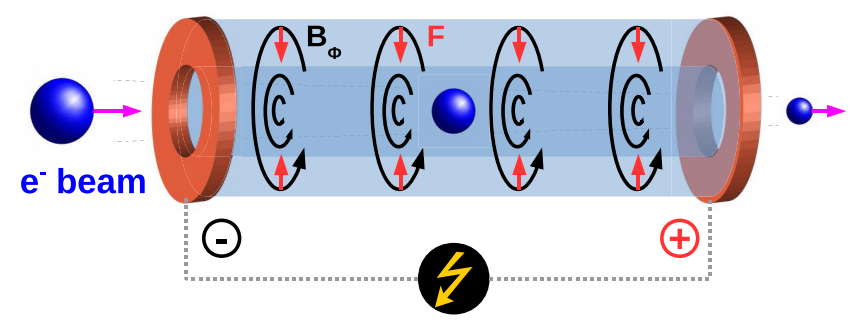

FIG. 3. Representation of the active-plasma lens working mechanism. A discharge current is applied to the capillary through two symmetric electrodes. The current generates an azimuthal magnetic field $\left(\mathbf{B}_{\phi}\right)$ that produces a focusing force $(\mathbf{F})$ for the incoming electron beam.

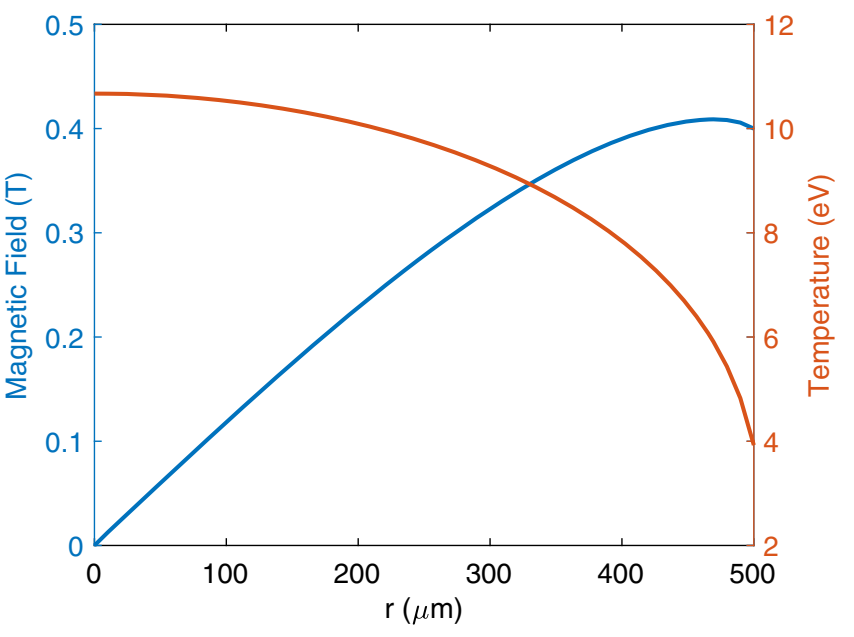

FIG. 4. Calculated profiles of the magnetic field (blue curve) and plasma temperature (red curve) along the capillary radius. The current discharge used for the calculation is $I_{D}=1 \mathrm{kA}$. The gas density is set to $n_{\text {gas }}=10^{16} \mathrm{~cm}^{-3}$.

initiated. In this case, the equilibrium is determined only by the balance between Ohmic heating and cooling due to the electron heat conduction. Figure 4 shows the resulting magnetic field and plasma temperature computed for a capillary with $R_{c}=500 \mu \mathrm{m}$ and a current discharge of $I_{D}=1 \mathrm{kA}$. The initial density of the neutral $\mathrm{H}_{2}$ gas is $n_{\text {gas }}=10^{16} \mathrm{~cm}^{-3}$. The nonlinearity of the magnetic field [dictated, according to Eq. (2), by a nonuniform current density $J(r)$ ] is one of the sources leading to the normalized emittance growth of the beam during focusing, although a recent work demonstrated the possibility to mitigate these effects [43].

\section{SYSTEM PERFORMANCES}

The extraction system we are discussing exploits the different focusing provided by an APL on beams with different energies [48]. This allows one to tune the APL to transport the witness through the collimator and, at the same time, overfocus the driver and remove its charge. A summary of the so-developed beam line is summarized in Table II.

TABLE II. Optimized parameters (size, radius, and position) for the APLs and collimator used in the proposed extraction system. The position of each element (along $z$ ) is relative to the exit of the PWFA module. For the APLs, the current discharge $\left(I_{D}\right)$ and plasma density $\left(n_{p}\right)$ are also reported.

\begin{tabular}{lccccc}
\hline \hline & Size $(\mathrm{cm})$ & Radius $(\mathrm{mm})$ & $z(\mathrm{~cm})$ & $I_{D}(\mathrm{kA})$ & $n_{p}\left(\mathrm{~cm}^{-3}\right)$ \\
\hline APL 1 & 2 & 0.5 & 15 & 1 & $10^{16}$ \\
Collimator & 3 & 0.2 & 97 & & \\
APL 2 & 1 & 0.5 & 135 & 0.6 & $10^{16}$ \\
\hline \hline
\end{tabular}




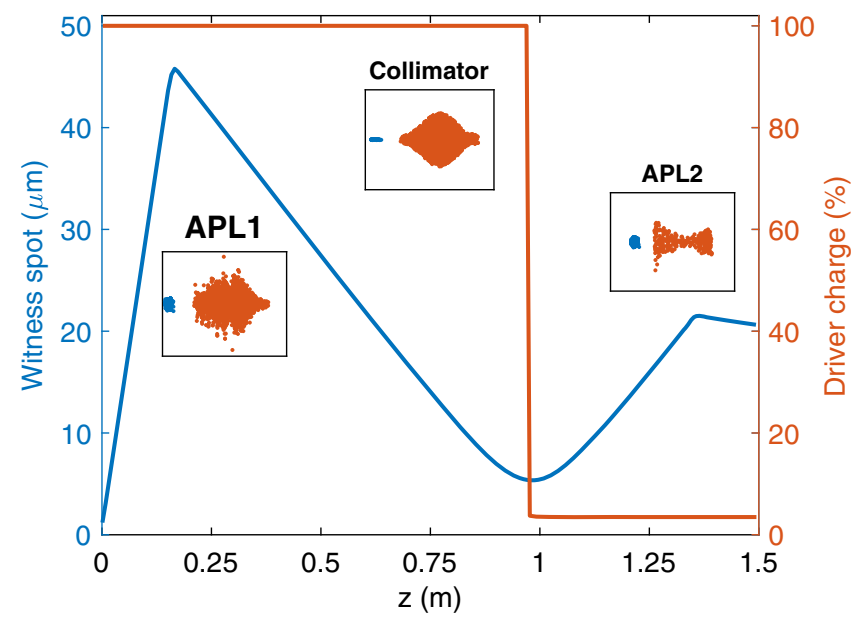

FIG. 5. Evolution of the witness envelope (blue line) and driver charge (red line) along the beam line. The insets show the $x-z$ plane of the bunches at the entrance of the first APL, the collimator, and the second APL.

Figure 5 shows the evolution of the witness envelope (blue line) and driver charge (red line) along the system, consisting of two APLs and a lead collimator between them. The system has been optimized on the LPS at the exit of the PWFA module, as the one reported in Fig. 1(a). The first lens consists of a 2-cm-long discharge capillary with $500 \mu \mathrm{m}$ hole radius. The focusing is obtained by applying a discharge current $I_{D}=1 \mathrm{kA}$. The position of the lens with respect to the PWFA module has been carefully chosen to preserve as much as possible the witness normalized emittance. On one side, according to Eq. (1), short drifts would be preferable to avoid emittance degradation due to the large divergence of the beam. On the other side, small drifts would produce a small witness spot at the APL entrance, i.e., a larger bunch density. As demonstrated in our previous work [43], large bunch densities produce nonnegligible plasma wakefields in the APL that, being nonlinear, would increase the beam emittance during the lens focusing. A trade-off is thus necessary to balance these two contributions. In Fig. 5, the best compromise has been found by moving the entrance on the first APL $15 \mathrm{~cm}$ downstream from the PWFA module. In this case, the normalized emittance increased in the drift from 0.6 to $0.7 \mu \mathrm{m}$ (rms).

The driver and witness are then transported to the entrance of the collimator whose radius is $R_{\text {coll }}=200 \mu \mathrm{m}$. Here the witness spot size is $\sigma_{w} \approx 10 \mu \mathrm{m}(\mathrm{rms})$, while the driver is almost a hundred times larger $\left(\sigma_{d} \approx 1 \mathrm{~mm}\right)$. In such a way, the majority of the driver charge $(\approx 98 \%)$ is removed by the collimator, with only $4 \mathrm{pC}$ that have remained after it; more details about the choice of the collimator aperture and particle interactions with its body are reported in Sec. V B. The system ends with the second APL ( $1 \mathrm{~cm}$ long with $I_{D}=0.6 \mathrm{kA}$ ) that refocuses the witness downstream from the collimator and allows for its transport through the rest of the beam line. Figure 6 shows the evolution of the witness



FIG. 6. Evolution of the witness normalized emittance along the beam line. The points where there is an increase of such a quantity are labeled accordingly.

normalized emittance along the beam line. As expected, the increase of such a quantity is foreseen in the initial drift downstream from the PWFA module [according to Eq. (1)] and in the two active-plasma lenses (more details are reported in Sec. IVA).

Considering all the elements and drift spaces involved, the total length of the system is $1.4 \mathrm{~m}$. In view of a compact facility like the one envisioned by the EuPRAXIA design study, such a small size is a key feature. Although the witness emittance doubles with respect to the one at the PWFA exit, the performances of the FEL are not affected. The lasing condition to fulfill is indeed $\epsilon_{g}=\epsilon_{n} / \gamma \leq$ $\lambda_{\mathrm{FEL}} / 4 \pi$ [49,50]; i.e., the geometric emittance $\epsilon_{g}$ must be smaller than the diffraction-limited photon beam $\left(\lambda_{\mathrm{FEL}} \approx 3 \mathrm{~nm}\right.$ for the case of interest). Regarding the amount of drive beam depletion, at the exit of the second APL we have 4 pC charge, more than $100 \mathrm{MeV}$ energy spread, $\approx 20 \mu \mathrm{m}$ emittance, and an equivalent current of $14 \mathrm{~A}$. We expect thus that such a residual beam would be barely transported through the subsequent beam lines, matched on the parameters of the witness beam. Following these results, the amount of driver charge eventually transported up to the FEL undulators is expected to be reasonably negligible.

\section{A. Beam dynamics in the active-plasma lenses}

As previously discussed, the focusing and guiding of an electron beam in an APL is actually due to the magnetic field generated by the discharge current, while the plasma acts only as a conducting medium. However, the dynamics of a beam is also affected by the interaction with the plasma that induces plasma wakefields acting on the beam itself [51-53]. In the limit of an electron beam with a density comparable to or smaller than the plasma one $\left(n_{b} \lesssim n_{p}\right)$, the wakefields can be described by using the $2 \mathrm{D}$ plasma 
wakefield theory in the linear regime [36]. If we rewrite the bunch density by separating the longitudinal and transverse profiles as $n_{b}(z, r)=n_{b, l}(z) \cdot n_{b, t}(r)$, the wakefields can be expressed as

$$
\begin{aligned}
W_{z}(z, r)= & \frac{q_{e}}{\epsilon_{0}} R(r) . \\
& \times \int_{-\infty}^{z} n_{b, l}\left(z^{\prime}\right) \cos \left(k_{p}\left(z-z^{\prime}\right)\right) d z^{\prime}, \\
W_{r}(s, r)= & \frac{q_{e}}{\epsilon_{0} k_{p}} \frac{d R(r)}{d r} . \\
& \times \int_{-\infty}^{z} n_{b, l}\left(z^{\prime}\right) \sin \left(k_{p}\left(z-z^{\prime}\right)\right) d z^{\prime},
\end{aligned}
$$

where $\epsilon_{0}$ is the vacuum permittivity and $q_{e}$ the electron charge. We have also introduced the plasma wave number $k_{p}$ and the function $R(r)$ defined as

$$
\begin{aligned}
R(r)= & k_{p}^{2} K_{0}\left(k_{p} r\right) \int_{0}^{r} n_{b, r}\left(r^{\prime}\right) I_{0}\left(k_{p} r^{\prime}\right) r^{\prime} d r^{\prime} \\
& +k_{p}^{2} I_{0}\left(k_{p} r\right) \int_{r}^{\infty} n_{b, r}\left(r^{\prime}\right) K_{0}\left(k_{p} r^{\prime}\right) r^{\prime} d r^{\prime},
\end{aligned}
$$

where $I_{0}$ and $K_{0}$ are the modified Bessel functions of zeroth and first order, respectively. From Eqs. (3) and (4), we can thus compute the forces $F_{z, r}=q_{e} W_{z, r}$ acting on the beam. As a consequence, we can see that, on the transverse plane, the plasma produced a net focusing. Indeed, when a relativistic electron bunch travels in a plasma, the spacecharge field of the electron beam is canceled by the plasma; thus, the beam is pinched by its own magnetic field. Such a mechanism happens in so-called passive plasma lenses, widely investigated in past years [54-56] and more recently [57].

Starting from this set of equations, we have developed a plasma simulation code that allows one to track the evolution of a test electron beam. Figure 7 shows a snapshot of the radial $\left(W_{r}\right)$ and longitudinal $\left(W_{z}\right)$ wakefields induced by the $30 \mathrm{pC}$ witness bunch moving in a plasma background with density $n_{p}=10^{16} \mathrm{~cm}^{-3}$. We can see that transverse (longitudinal) fields as large as $5(30) \mathrm{MV} / \mathrm{m}$ are produced. While the longitudinal dynamics is not affected, the transverse evolution is influenced by both the transverse plasma wakefield $W_{r}$ and the azimuthal magnetic field $B_{\text {ext }}$ induced by the discharge current. Figure 8 shows the evolution of the witness normalized emittance and envelope along the two APLs used in the setup reported in Table II. For the sake of simplicity, we have not considered any plasma ramp at the entrance and exit of the lens, resulting in a a sharp transition between the vacuum and the plasma channel. As shown, most of the emittance growth happens in the first (and longer) lens, where the witness transverse spot size is larger (43 $\mu \mathrm{m} \mathrm{rms)} \mathrm{with} \mathrm{respect} \mathrm{to} \mathrm{the} \mathrm{one} \mathrm{entering} \mathrm{into} \mathrm{the}$



FIG. 7. Simulated radial (top) and longitudinal (bottom) plasma wakefield produced into the APL by the witness bunch. The red dashed ellipse shows the rms size of the witness bunch.

second lens ( $23 \mu \mathrm{m} \mathrm{rms})$. Such growth is due to both the nonlinearities of the $B_{\text {ext }}$ field (cf. Fig. 4) and $W_{r}$.

To better understand the dynamics during the lensing effect, we can split the two effects involved, i.e., the passive lensing due to the induced plasma wakefields and the active lensing due to the magnetic field produced by the discharge current. Figure 9 shows a comparison for the same two parameters, normalized emittance and spot, with (solid lines) and without (dashed lines) the external magnetic field in the first APL. We can see that without the dischargeinduced magnetic field there is almost no focusing (unperturbed growth of the beam spot size), but, in spite of that, the emittance increases due to the nonlinear force exerted by the transverse plasma wakefields. The increase of emittance due to the passive lensing (i.e., $W_{r}$ ) and the external field is approximately the same, i.e., $\Delta \epsilon_{n} \approx 0.2 \mu \mathrm{m}$. This scenario,
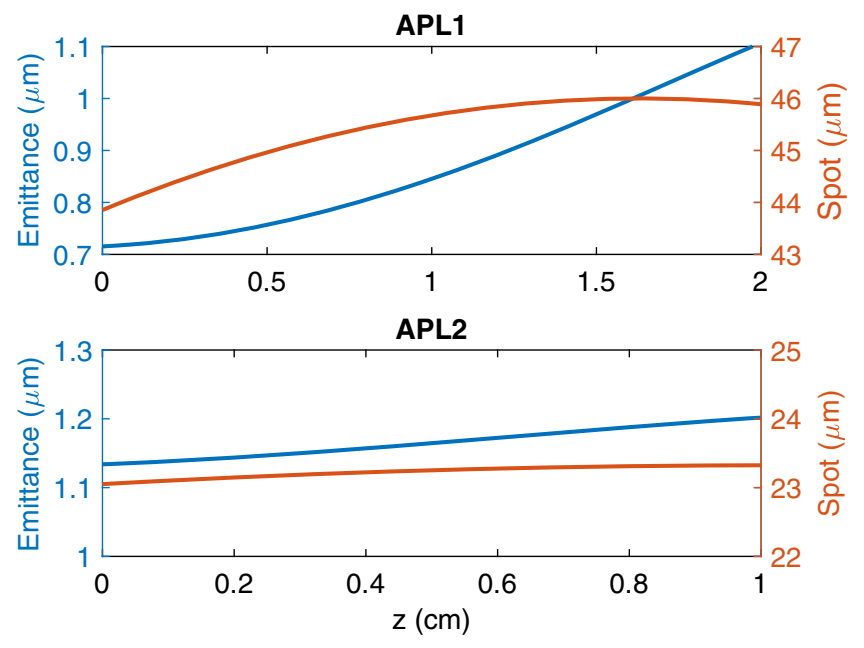

FIG. 8. Evolution of the witness normalized emittance (blue lines) and spot size (red lines) along the two APLs. 


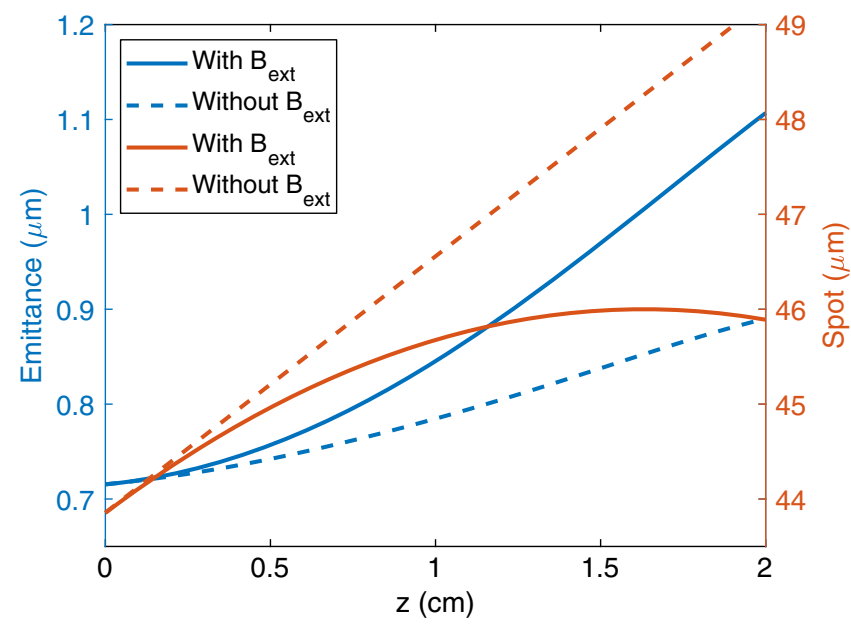

FIG. 9. Witness normalized emittance (blue lines) and spot size (red lines) along a 2-cm-long APL by including (solid lines) or not (dashed lines) the magnetic field produced by the discharge current.

however, represents the best compromise, since smaller (larger) witness spot sizes would enhance the contribution due to the $W_{r}\left(B_{\text {ext }}\right)$ term and produce an overall larger emittance degradation [58].

\section{B. Parametric study}

The tunability of the system for different beam parameters is ensured by simply changing the discharge current applied to the APLs in order to adjust the witness focusing and transport along the beam line. Once the transport optics is optimized on the witness side, the disposal of the driver charge mainly depends on its own 6D phase space and can be maximized by properly tailoring the collimator geometry (aperture and length). In this section, we discuss the dynamics of the driver bunch for different configurations, while the witness bunch is assumed to be as in the reference case described so far. This allows us to not change the beam line optics and check how it reacts in the presence of the incoming driver.

Figure 10(a) shows how the removal of the charge is affected according to the incoming beam energy. The system is matched on the witness, so we expect that for energies close to the witness one $(\approx 1 \mathrm{GeV})$ the removal of the particles is less effective. We see that such a charge selection is basically obtained: The lower the beam energy, the larger the amount of charge cut by the system. On the contrary, at larger energies, the system handles the driver beam as in the case of the witness, so less charge is removed. Another parameter that could change downstream from the PWFA module is the bunch normalized emittance that, actually, determines its divergence. Considering the driver bunch, its divergence at the PWFA exit strongly affects the effective charge collimation. Figure 10(b) shows how the driver charge is dependent on such a parameter. As expected, larger beam divergences
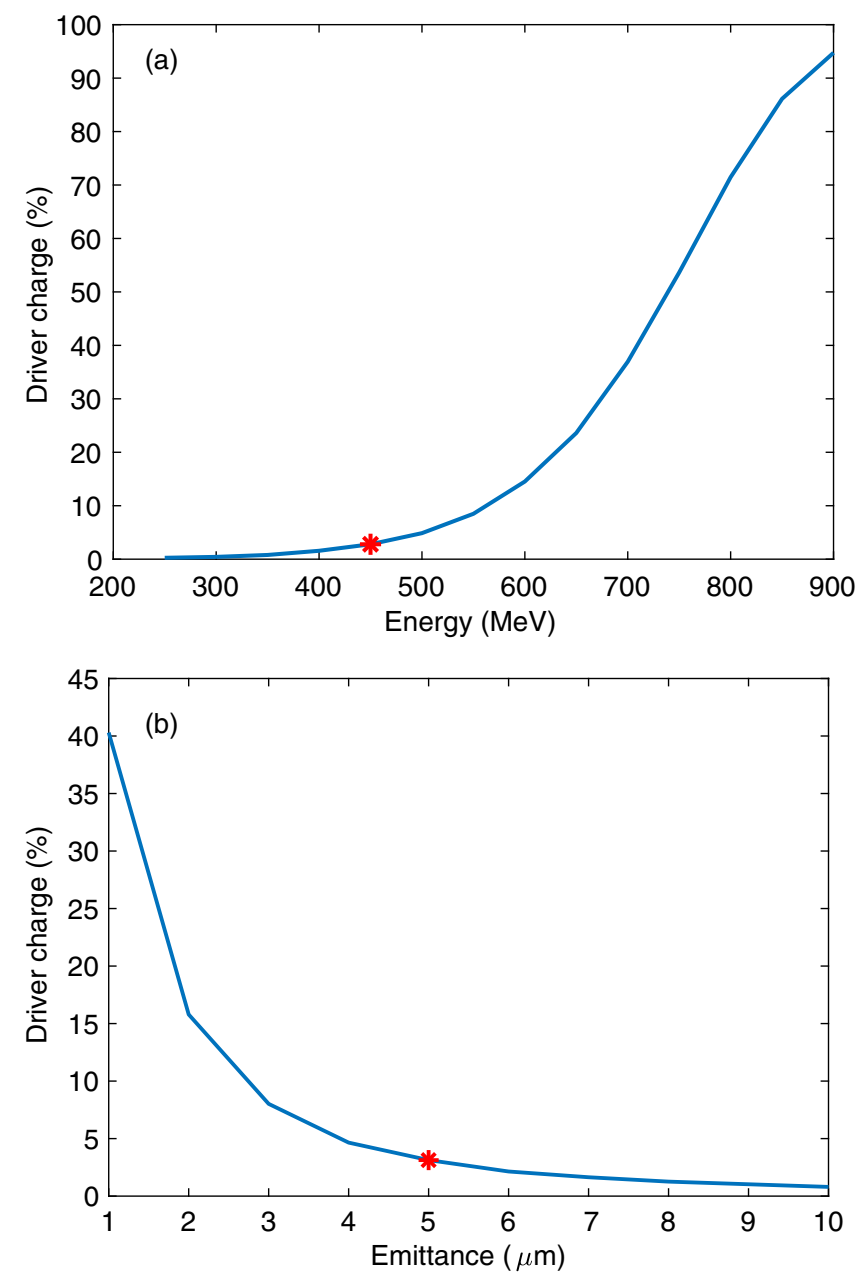

FIG. 10. Driver charge at the end of the system for different driver energies (a) and normalized emittances (b). The red asterisks show the reference case described in Table I.

(due to larger emittances) are favorable, since the beam would reach the collimator with a larger transverse spot with more particles interacting with it and, thus, removed. On the contrary, low emittances produce a more collimated beam and, in turn, a decrease of the charge cut operated by the collimator, since fewer particles hit its walls. In conclusion, the parametric study demonstrates that the system can be tuned on a particular configuration. For the sake of completeness, we have also repeated this analysis by changing the driver spot size and energy spread, but these parameters only slightly affect the removal of charge operated by the system (less than 10\% with respect to the reference case described so far) and have been omitted in this treatment.

\section{BEAM INTERACTIONS WITH THE COLLIMATOR}

\section{A. Wake potentials}

The interaction between the bunch electric field and the conductive walls of the lead collimator described so far is 
able to generate wakefields that can affect both the longitudinal and transverse dynamics of the traveling bunch, hindering the emittance preservation [59]. The electromagnetic interaction of charged particles with any surrounding environment can be quantified by solving the Maxwell equation to find the resulting electric and magnetic fields and then estimating the effects of these fields on the particle motion $[60,61]$. Assuming that the $z$ axis is the symmetry axis of the system under analysis and considering two particles that move along it, the electromagnetic field generated by a leading charge $q_{l}$ (located at $z=c t$ with a transverse offset $r_{1}$ with respect to the $z$ axis) produces a change of the momentum $\Delta \mathbf{p}$ on a trailing particle with charge $q_{t}$ (located behind the first one at $s=c t-z$ with a transverse offset $r_{2}$ ). The beam dynamics being different in the longitudinal and transverse directions, it is useful to separate the longitudinal momentum $\Delta p_{z}$ from the transverse component $\Delta \mathbf{p}_{\perp}$ and introduce the longitudinal and transverse wake functions [61] as, respectively,

$$
\begin{aligned}
w_{z}\left(r_{1}, r_{2}, s\right) & =\frac{c \Delta p_{z}}{q_{l} q_{t}}, \\
\mathbf{w}_{\perp}\left(r_{1}, r_{2}, s\right) & =\frac{c \Delta \mathbf{p}_{\perp}}{q_{l} q_{t}} .
\end{aligned}
$$

Regarding the longitudinal wake, if the $r_{1,2}$ offsets are small in comparison to the aperture of the pipe, we can remove the radial dependence and approximate $w_{z}(s)=w_{z}(0,0, s)$. For a bunch with longitudinal charge distribution $\rho(s)$, the wake potential $W_{z}(s)$ (the voltage lost for a test particle at position $s$ ) is thus given by

$$
W_{z}(s)=\int_{0}^{\infty} w\left(s^{\prime}\right) \rho\left(s-s^{\prime}\right) d s^{\prime} .
$$

In a similar way, by assuming $r_{1}=r_{2}=r$, the transverse wake potential $W_{\perp}(s)$ (that represents the transverse kick for a test particle at position $s$ ) can be approximated to its lowest-order linear term $\left[\mathbf{w}_{\perp}(r, r, s) \approx \mathbf{w}_{\perp}(s) / r\right]$ and expressed as

$$
W_{\perp}(s)=\frac{1}{r} \int_{0}^{\infty}\left|\mathbf{w}_{\perp}\left(s^{\prime}\right)\right| \rho\left(s-s^{\prime}\right) d s^{\prime} .
$$

The average of the longitudinal wake potential from Eq. (8) gives the loss factor $k_{l}$, while the average of the transverse wake from Eq. (9) represents the kick factor $k_{t}$.

From the previous equations, we thus have that the longitudinal wakes cause an energy decrease of the bunch while the transverse ones can affect the design orbit of the beam. To quantify the effects of these wakes on the witness bunch, we performed a numerical 3D simulation with the CST code [62]. The wake amplitude being proportional to the bunch charge, here we neglect the wakes induced by the driver, since the amount of its charge propagating through the collimator aperture is very small and, thus, not able to generate a significant wakefield that could affect the witness

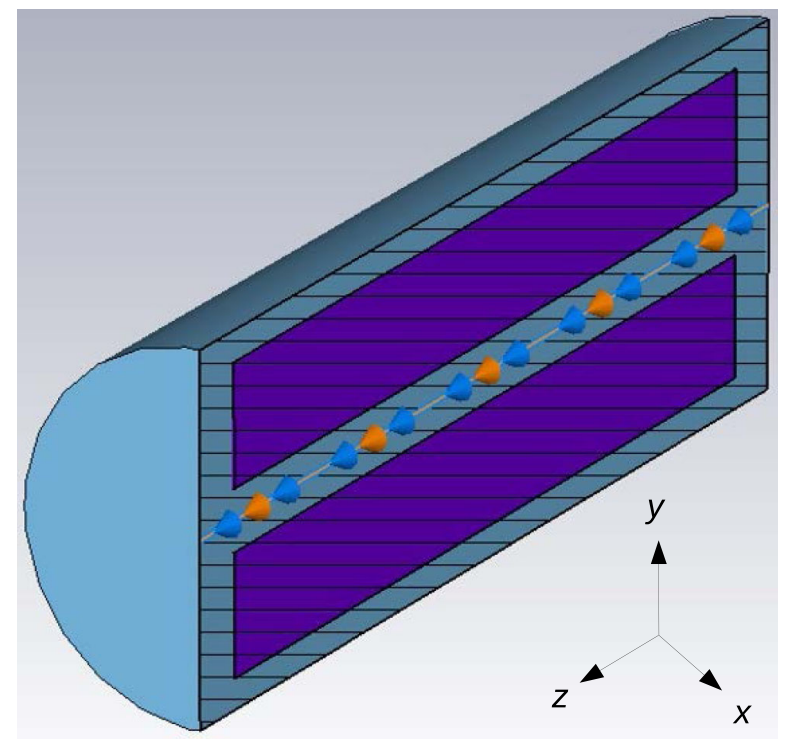

FIG. 11. Simulated collimator structure in CST. The beam simulated to generate and probe the wake potentials has the same properties of the witness bunch so far described.

dynamics. Figure 11 shows the simulated collimator structure. The collimator aperture is $400 \mu \mathrm{m}$ in diameter and $3 \mathrm{~cm}$ in length. The simulated beam, used to generate and probe at the same time as the induced wake potentials, has the same properties of the witness bunch so far described. Both longitudinal $W_{z}$ and transverse $W_{y}$ wake potentials are calculated along the axis and with a $\Delta y=50 \mu \mathrm{m}$ transverse offset, respectively. They are shown in Fig. 12 together with the witness bunch distribution. From the simulations, we obtained a longitudinal loss factor $k_{l}=1.65 \mathrm{kV} / \mathrm{pC}$ and a kick factor $k_{t}=1.92 \mathrm{~V} / \mathrm{pC} / \mathrm{mm}$. From these loss

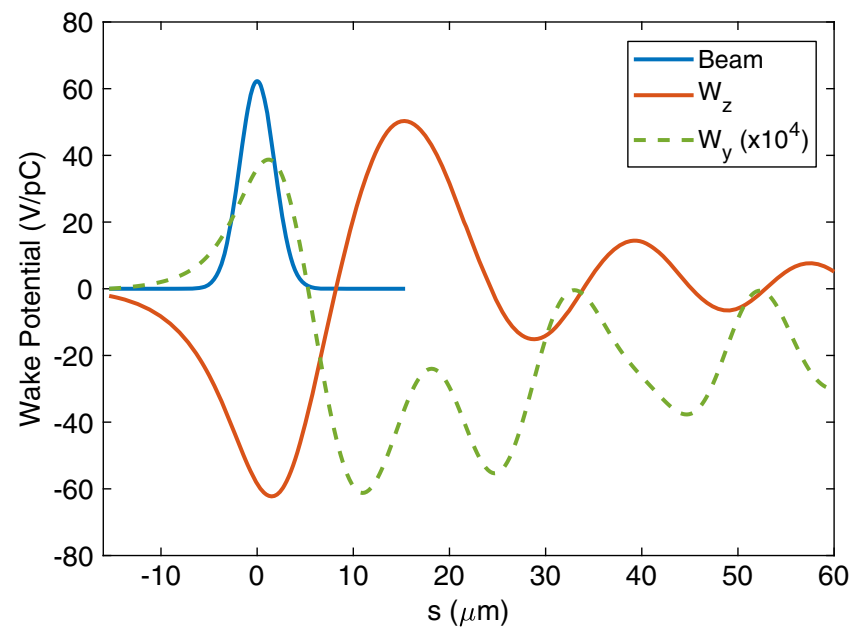

FIG. 12. Longitudinal (red curve) and transverse (green curve) wake potentials induced into the collimator by the witness bunch (blue curve). To represent the lines with the same axis scale, the transverse wake $W_{y}$ (calculated at distance $r=50 \mu \mathrm{m}$ from the collimator axis) has been enhanced by a factor of $10^{4}$. 
parameters, it is then possible to derive the energy decrease and the transverse kick received by the bunch. The energy loss can be easily computed as $\Delta E=k_{l} \cdot Q \approx 50 \mathrm{keV}$. Similarly, the angular deflection produced by the transverse wake on a beam with energy $E$ can be estimated as [63] $\Delta \theta=$ $\Delta y \cdot k_{t} \cdot Q / E \approx 2.88 \times 10^{-9} \mathrm{rad}$. From the resulting numbers, we can thus conclude that the influence of the wakefields produced into the collimator by the witness bunch does not affect its dynamics and can be neglected.

\section{B. Particle-matter interactions}

The interaction of the driver beam with the lead collimator has been numerically simulated by means of the GEANT4 framework, a single-particle tracker which takes into account all the fundamental radiation-matter interactions [64]. GEANT4 is a toolkit for simulating the passage of particles through matter. It includes many functionalities like tracking, geometry, physics models, and hits. Many physics processes are included and cover a wide range of interactions like electromagnetic, hadronic, and optical processes, a large set of long-lived particles, materials, and elements, over a wide energy range (from hundreds of $\mathrm{eV}$ up to $\mathrm{TeV}$ ). In defining and implementing all the involved components, all aspects of the simulation process have been included: (i) the geometry of the collimator system, (ii) the material involved (lead), (iii) the fundamental particles of interest (electrons), (iv) the generation of primary particles of events (electrons, hadrons, and photons), (v) the tracking of particles through materials, and (vi) the physics processes governing particle interactions (bremsstrahlung, pair production, multiple scattering, etc.).

For the current simulation, we proceeded as in the following. First, we transported with GPT the beam exiting from the first APL up to the collimator entrance. Here we imported and converted the GPT bunch in order to be treated with GEANT4. Finally, the GEANT4 simulation output is imported again in GPT and used as input for the second APL. In the GEANT4 simulation the FTFP_BERT physics list has been adopted; it contains the standard electromagnetic and hadronic interactions, the latter ones implemented using the FTF parton string and Bertini cascade models [65]. The performances of the collimator have been optimized by varying four parameters in the simulation: the thickness, the inner and outer radii, and the distance of the collimator from the first APL. The final configuration reported in Table II represents the best compromise regarding the driver dumping and the preservation of the witness beam charge. It consists of a $L_{\text {coll }}=3$-cm-long lead cylinder with outer radius $R_{\text {coll }}=1 \mathrm{~cm}$ and $R_{h}=$ $200 \mu \mathrm{m}$ radius aperture. According to the GEANT4 simulation, such a solution allows one to remove $\approx 98 \%$ of the driver charge while the witness remains untouched. Figure 13 shows the resulting tracks of the primary and secondary particles produced during the interaction of the driver bunch with the collimator.

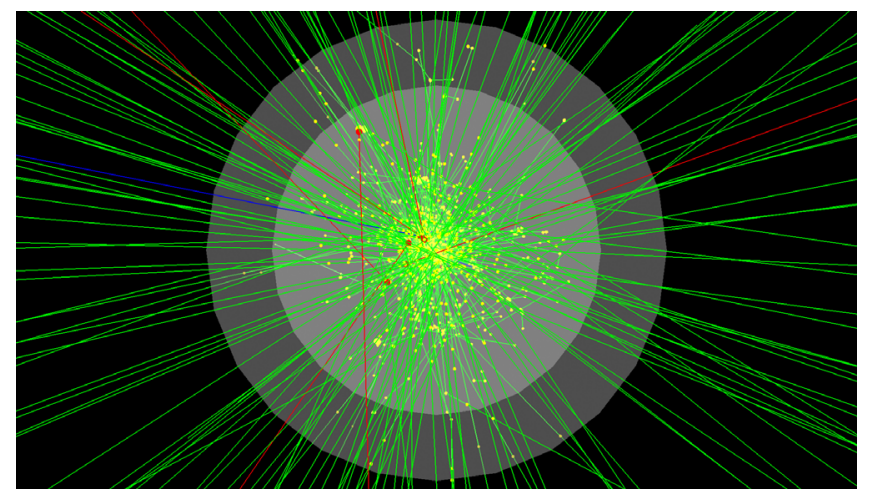

FIG. 13. Driver beam interaction with the collimator. The colors of the tracks stand for electrons (red), positrons (blue), and photons (green) in the $\gamma$ - and $\mathrm{x}$-ray range.

The simulation recorded all the scattered and emitted particles after the interaction. Most of the deposited energy is converted into electrons and photons. Figure 14 shows the
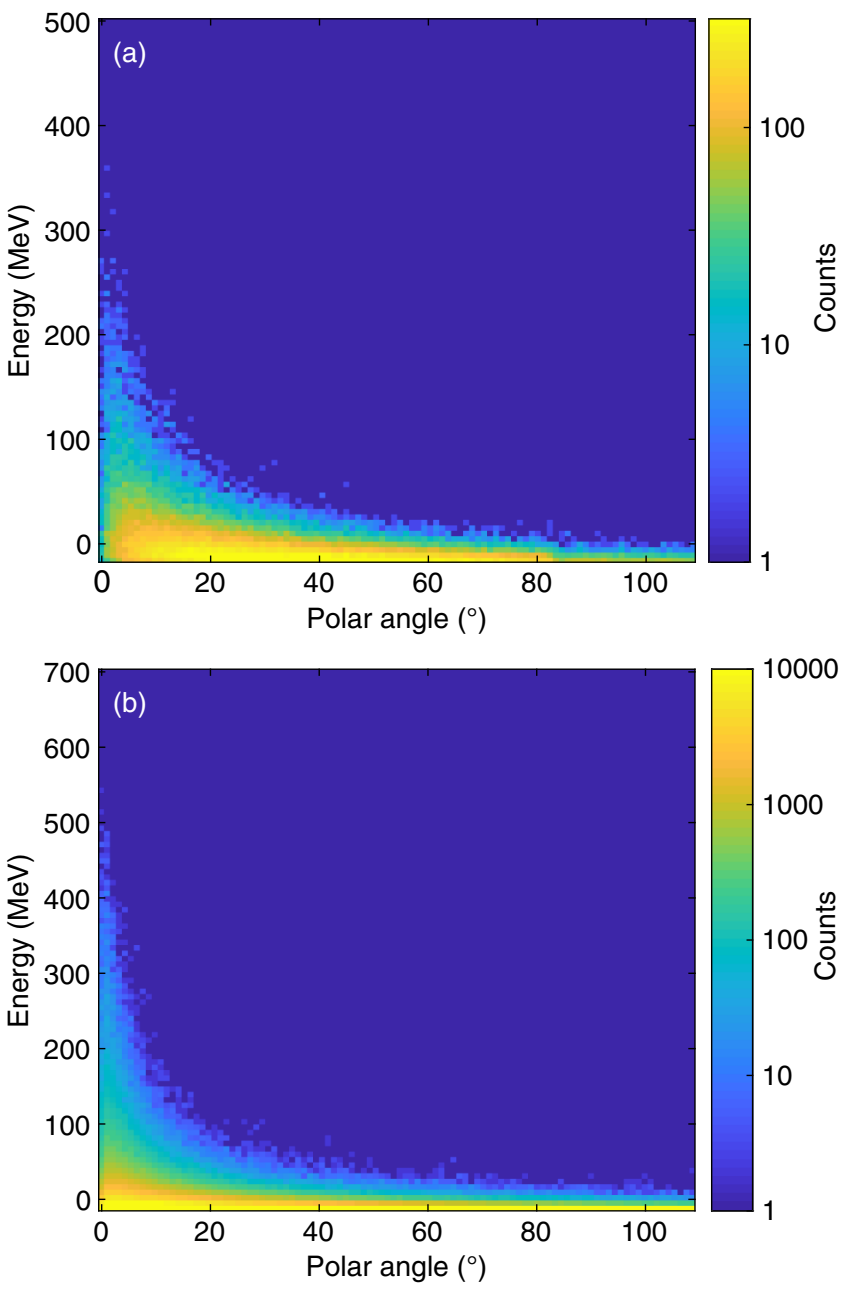

FIG. 14. Polar angular distribution as a function of the energy for the electrons (a) and photons (b) produced after the interaction. On the $x$ axis, angles close to $0^{\circ}\left(180^{\circ}\right)$ mean that the particles are moving forward (backward) with respect to the incoming electron beam. 

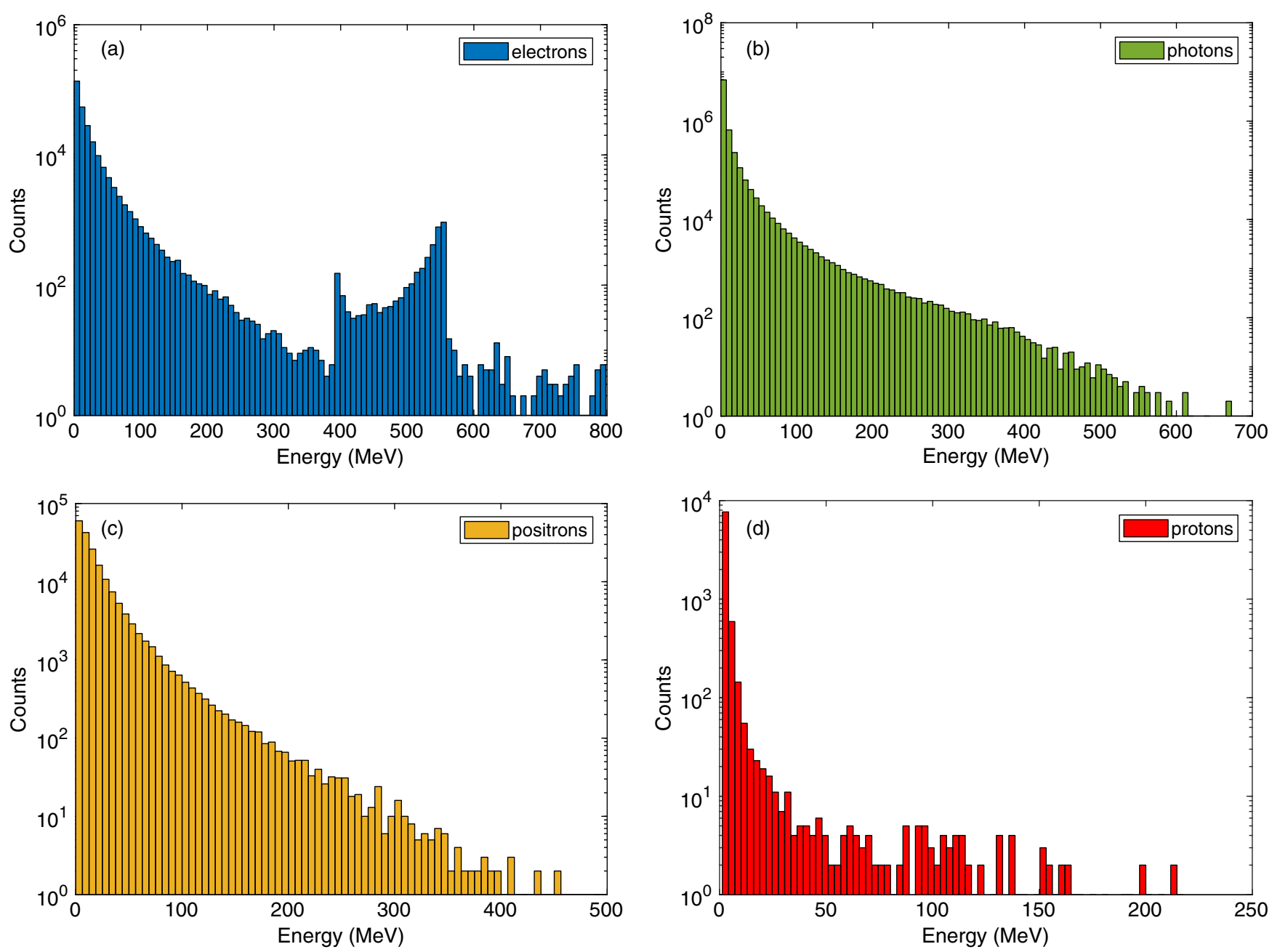

FIG. 15. Energy spectrum of the scattered and emitted electrons (a), photons (b), positrons (c), and protons (d) from the collimator after the interaction with the incoming electron beam. The particles have been collected over a $4 \pi$ solid angle around the collimator.

polar angular distribution ( $\theta$ angle with respect to the $z$ axis, representing the incoming beam direction) as a function of the energy for these two kinds of particle. The plots highlight that the ones carrying the largest energies are emitted forward $(\theta \approx 0)$, while only low-energy particles move radially $\left(\theta \approx 90^{\circ}\right)$, that is, perpendicular to the incoming beam. Finally, in Fig. 15, we show the overall counts for not only electrons and photons but also positrons and protons. As we can see, a large number of $\gamma$ or $\mathrm{x}$ rays are produced, up to $10^{7}$ by assuming $3 \times 10^{5}$ incoming electrons. This is a potential source of background for any detector or diagnostics installed around the collimator that, thus, would require a proper shielding system to be adopted for any practical purpose.

\section{Temperature analysis}

A final remark regards the total energy deposition and temperature rise in the lead collimator. This is important information that allows one to estimate the maximum repetition rate $f_{\text {rep }}$ the system can sustain. With the beam configuration discussed so far, the maximum energy deposited for each shot is $E_{\text {beam }} \approx 0.1 \mathrm{~J}$, corresponding to a beam power $P_{\text {beam }}=f_{\text {rep }} \cdot E_{\text {beam }}$. For the sake of simplicity, we have assumed that all the driver particles are absorbed and neglected the escaping ones after the interaction; i.e., we are investigating the worst situation where the largest temperature increase is reached.

To determine the temperature evolution, we must solve the heat flow equation [66]

$$
\rho c_{p} \frac{d T}{d t}=\kappa_{c} \frac{1}{r} \frac{d}{d r}\left(r \frac{d T}{d r}\right)+q_{\text {beam }}-h_{\mathrm{rad}},
$$

with boundary conditions $T\left(t_{0}, r\right)=T\left(t, R_{\text {coll }}\right)=T_{0}$ and $T_{0}=300 \mathrm{~K}$ the room temperature. In Eq. (10), we have that $\rho \approx 11.34 \mathrm{~g} \mathrm{~cm}^{-3}$ is the lead density, $c_{p} \approx$ $128 \mathrm{~J} \mathrm{Kg}^{-1} \mathrm{~K}^{-1}$ its specific heat, and $\kappa_{c} \approx 34.7 \mathrm{~W} \mathrm{~m}^{-1} \mathrm{~K}$ its thermal conductivity. The three terms on the right side of the equation represent (i) the heat rate through conduction; (ii) the net rate of heat generation given by the incoming 
particle beam; and (iii) the heat rate lost by radiation. All the terms have physical units of power per volume $\left(\mathrm{W} \mathrm{m}^{-3}\right)$. At the thermal equilibrium, the temperature reached by the collimator during beam irradiation can be calculated by equating to zero the term on the left side of Eq. (10). Assuming an electron beam with a Gaussian transverse profile and radius $\sigma_{r}(\approx 1 \mathrm{~mm}$ for the driver bunch at the entrance of the collimator; see Sec. IV), the $q_{\text {beam }}$ term can be expressed as

$$
q_{\text {beam }}=\frac{P_{\text {beam }}}{2 \pi \sigma_{r}^{2} L_{c}} \exp \left(-\frac{r^{2}}{2 \sigma_{r}^{2}}\right) .
$$

The heat rate loss due to radiation is given by the wellknown Stefan-Boltzmann law

$$
h_{\mathrm{rad}}=\frac{\varepsilon}{L_{c}} \sigma_{S B}\left(T^{4}-T_{0}^{4}\right) \text {, }
$$

where $\varepsilon \approx 0.07$ is the lead emissivity [67] and $\sigma_{S B}$ is the Stefan-Boltzmann constant. Since the energy deposition on the lead collimator produces an expansion of its size, we evaluate the maximum beam repetition rate by fixing the maximum tolerable variation along the radial dimension to $\Delta R_{h} / R_{h}=10 \%\left(\Delta R_{h}=20 \mu \mathrm{m}\right.$ in our case $)$. By knowing that the lead linear thermal expansion coefficient is $\alpha_{c} \approx$ $29 \times 10^{-6} \mathrm{~K}^{-1}$ [68], the change of its radial size can be calculated as [69]

$$
\Delta R=\alpha_{c} \int r \cdot d T(r)=\alpha_{c} \int r \cdot \nabla T(r) \cdot d r,
$$

where $\nabla T(r)=d T(r) / d r$ is the radial gradient of the temperature.

We found the $\Delta R_{h}$ elongation is reached for a maximum repetition rate $f_{\text {rep }} \approx 5 \mathrm{kHz}$, corresponding to an input power $P_{\text {beam }} \approx 500 \mathrm{~W}$. Figure 16(a) shows the temporal evolution of the collimator temperature calculated at $r=R_{h}$. The plot highlights that the thermal equilibrium is reached after of $t \approx 3 \mathrm{~s}$ irradiation time. The temperature increased by $\Delta T \approx 170 \mathrm{~K}$; i.e., it reached the absolute temperature of $470 \mathrm{~K}$. The lead melting point $(\approx 600 \mathrm{~K}$ [70]) is reached only with $f_{\text {rep }} \approx 9 \mathrm{kHz}$. The radial trend of the temperature is shown in Fig. 16(b). As expected, larger temperatures are produced at small radii, close to the beam propagation axis. The temperature then decreases toward the collimator walls. These results point out that larger (smaller) input powers would produce a larger (smaller) temperature increase and, in turn, a larger (smaller) deformation of the collimator aperture. The calculation we presented suggests that the proposed system is fully compatible with normal-conducting accelerator facilities operating at frequencies of $10-100 \mathrm{~Hz}$, like the one envisioned by the EuPRAXIA design study. On the contrary, for superconducting accelerators working with
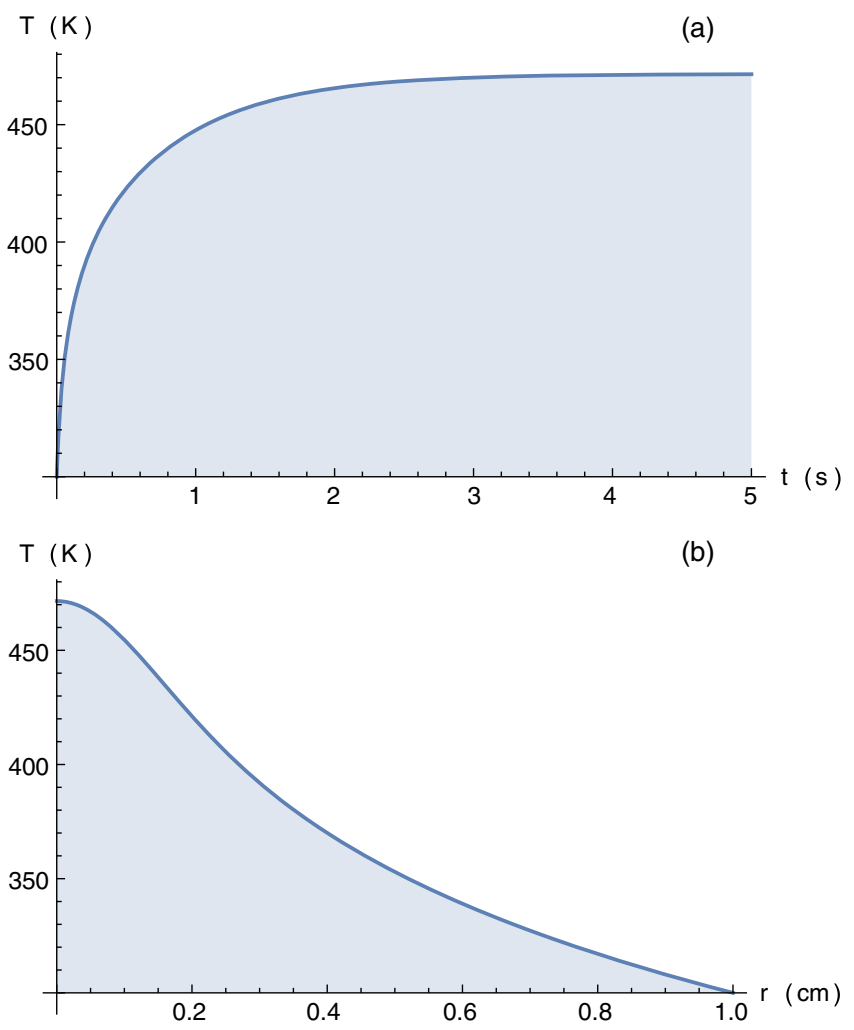

FIG. 16. (a) Temporal evolution of the collimator temperature (computed at radial position $r=R_{h}$ ) during the interaction with the driver beam. (b) Radial evolution of the collimator temperature computed at time $t=5 \mathrm{~s}$.

megahertz repetition rates, the collimator should be assisted by a proper refrigerator system.

\section{CONCLUSIONS}

The beam-driven plasma wakefield acceleration technique represents one of the best candidates to develop nextgeneration compact accelerators. Being a new technology, it must solve several issues in order to be adopted for any practical use such as user-oriented applications. As highlighted in this work, a drawback is represented by the removal of the high-charge and energy-depleted driver bunch and, at the same time, the need to provide an efficient capture of the witness bunch avoiding its normalized emittance degradation. Here we have presented a possible solution, based on the use of active-plasma lenses, that has three main key features: (i) the tunability offered by the lenses themselves that allow one to adapt the system to different configurations; (ii) a good dumping efficiency of the driver bunch, with only few percent of the incoming driver beam that remained at the end of the transport chain; and (iii) the compactness of the entire solution, less than $1.4 \mathrm{~m}$, for the manipulation of $\mathrm{GeV}$-class beams. For the scenario we have chosen as reference, i.e., the one represented by a proposed facility based on the EuPRAXIA design study, we have demonstrated that, 
downstream from the PWFA booster module, the accelerated $1 \mathrm{GeV}$ witness can be efficiently captured and handled (in view of its transport up to the FEL undulators and user stations) with its normalized emittance that grew from 0.6 to $1.2 \mu \mathrm{m}$ at the end of the extraction system. This number can be further optimized (and emittance better preserved) by implementing, for instance, plasma lenses with properly shaped density profiles [14,71]. At the same time, we showed that a lead collimator located between the two plasma lenses is a cost-effective solution that allows one to dump the $200 \mathrm{pC}$ driver bunch to the level of few $\mathrm{pC}$ without affecting the witness. The study of the collimator, in particular, has been conducted by analyzing both the resistive wakefields excited along its aperture and the particle-matter interactions with the collimator walls. The results confirmed that such a solution can be implemented in a future facility based on plasma acceleration where the compactness represents one of the main goals.

\section{ACKNOWLEDGMENTS}

This work has been partially supported by the EU Commission in the Seventh Framework Program, Grant Agreement No. 312453-EuCARD-2, the European Union Horizon 2020 research and innovation program, Grant Agreement No. 653782 (EuPRAXIA), and the PLADIP project funded by the INFN GRANT_73.

[1] T. Tajima and J. M. Dawson, Laser Electron Accelerator, Phys. Rev. Lett. 43, 267 (1979).

[2] W. Leemans, B. Nagler, A. Gonsalves, C. Tóth, K. Nakamura, C. Geddes, E. Esarey, C. Schroeder, and S. Hooker, GeV electron beams from a centimetre-scale accelerator, Nat. Phys. 2, 696 (2006).

[3] J. Faure, C. Rechatin, A. Norlin, A. Lifschitz, Y. Glinec, and V. Malka, Controlled injection and acceleration of electrons in plasma wakefields by colliding laser pulses, Nature (London) 444, 737 (2006).

[4] I. Blumenfeld, C. E. Clayton, F.-J. Decker, M. J. Hogan, C. Huang, R. Ischebeck, R. Iverson, C. Joshi, T. Katsouleas, N. Kirby, W. Lu, K. A. Marsh, W. B. Mori, P. Muggli, E. Oz, R. H. Siemann, D. Walz, and M. Zhou, Energy doubling of $42 \mathrm{GeV}$ electrons in a metre-scale plasma wakefield accelerator, Nature (London) 445, 741 (2007).

[5] W. Lu, W. An, C. Huang, C. Joshi, W. B. Mori, M. Hogan, T. Raubenheimer, A. Seryi, P. Muggli, and T. Katsouleas, High transformer ratio PWFA for application on XFELs, Proceedings of PAC09, Vancouver, BC, Canada, https:// accelconf.web.cern.ch/AccelConf/PAC2009/papers/ we6rfp098.pdf

[6] M. Litos, E. Adli, W. An, C. Clarke, C. Clayton, S. Corde, J. Delahaye, R. England, A. Fisher, J. Frederico et al., High-efficiency acceleration of an electron beam in a plasma wakefield accelerator, Nature (London) 515, 92 (2014).
[7] A. R. Rossi, A. Bacci, M. Belleveglia, E. Chiadroni, A. Cianchi, G. Di Pirro, M. Ferrario, A. Gallo, G. Gatti, C. Maroli et al., The External-Injection experiment at the SPARC_LAB facility, Nucl. Instrum. Methods Phys. Res., Sect. A 740, 60 (2014).

[8] S. Steinke, J. Van Tilborg, C. Benedetti, C. Geddes, C. Schroeder, J. Daniels, K. Swanson, A. Gonsalves, K. Nakamura, N. Matlis et al., Multistage coupling of independent laser-plasma accelerators, Nature (London) 530, 190 (2016).

[9] J. Faure, Y. Glinec, A. Pukhov, S. Kiselev, S. Gordienko, E. Lefebvre, J.-P. Rousseau, F. Burgy, and V. Malka, A laserplasma accelerator producing monoenergetic electron beams, Nature (London) 431, 541 (2004).

[10] C. G. R. Geddes, C. Toth, J. van Tilborg, E. Esarey, C. B. Schroeder, D. Bruhwiler, C. Nieter, J. Cary, and W. P. Leemans, High-quality electron beams from a laser wakefield accelerator using plasma-channel guiding, Nature (London) 431, 538 (2004).

[11] E. Esarey, P. Sprangle, J. Krall, and A. Ting, Overview of plasma-based accelerator concepts, IEEE Trans. Plasma Sci. 24, 252 (1996).

[12] P. A. Walker, P. Alesini, A. Alexandrova, M. P. Anania, N. Andreev, I. Andriyash, A. Aschikhin, R. Assmann, T. Audet, A. Bacci et al., Horizon 2020 EuPRAXIA design study, J. Phys. Conf. Ser. 874, 012029 (2017).

[13] C. M. Sears, A. Buck, K. Schmid, J. Mikhailova, F. Krausz, and L. Veisz, Emittance and divergence of laser wakefield accelerated electrons, Phys. Rev. Accel. Beams 13, 092803 (2010).

[14] I. Dornmair, K. Floettmann, and A. Maier, Emittance conservation by tailored focusing profiles in a plasma accelerator, Phys. Rev. Accel. Beams 18, 041302 (2015).

[15] M. Migliorati, A. Bacci, C. Benedetti, E. Chiadroni, M. Ferrario, A. Mostacci, L. Palumbo, A. Rossi, L. Serafini, and P. Antici, Intrinsic normalized emittance growth in laser-driven electron accelerators, Phys. Rev. Accel. Beams 16, 011302 (2013).

[16] R. Pompili, M. Anania, M. Bellaveglia, A. Biagioni, F. Bisesto, E. Chiadroni, A. Cianchi, M. Croia, A. Curcio, D. Di Giovenale et al., Beam manipulation with velocity bunching for PWFA applications, Nucl. Instrum. Methods Phys. Res., Sect. A 829, 17 (2016).

[17] J. Van Tilborg, S. Steinke, C. Geddes, N. Matlis, B. Shaw, A. Gonsalves, J. Huijts, K. Nakamura, J. Daniels, C. Schroeder et al., Active Plasma Lensing for Relativistic Laser-Plasma-Accelerated Electron Beams, Phys. Rev. Lett. 115, 184802 (2015).

[18] R. Pompili, M. Anania, M. Bellaveglia, A. Biagioni, S. Bini, F. Bisesto, E. Brentegani, G. Castorina, E. Chiadroni, A. Cianchi et al., Experimental characterization of active plasma lensing for electron beams, Appl. Phys. Lett. 110, 104101 (2017).

[19] E. Chiadroni, M. Anania, M. Bellaveglia, A. Biagioni, F. Bisesto, E. Brentegani, F. Cardelli, A. Cianchi, G. Costa, D. Di Giovenale et al., Overview of plasma lens experiments and recent results at SPARC_LAB, Nucl. Instrum. Methods Phys. Res., Sect. A 909, 16 (2018).

[20] J. van Tilborg, S. Barber, H.-E. Tsai, K. Swanson, S. Steinke, C. Geddes, A. Gonsalves, C. Schroeder, E. Esarey, 
S. Bulanov et al., Nonuniform discharge currents in active plasma lense, Phys. Rev. Accel. Beams 20, 032803 (2017).

[21] R. Pompili, G. Castorina, M. Ferrario, A. Marocchino, and A. Zigler, Guiding of charged particle beams in curved capillary-discharge waveguides, AIP Adv. 8, 015326 (2018).

[22] EuPRAXIA design study, http://www.eupraxia-project.eu/ home.html.

[23] A. F. Pousa, A. M. de la Ossa, R. Brinkmann, and R. Assmann, Compact Multistage Plasma-Based Accelerator Design for Correlated Energy Spread Compensation, Phys. Rev. Lett. 123, 054801 (2019).

[24] M. Ferrario, D. Alesini, M. Anania, M. Artioli, A. Bacci, S. Bartocci, R. Bedogni, M. Bellaveglia, A. Biagioni, F. Bisesto et al., EuPRAXIA@ SPARC_LAB Design study towards a compact FEL facility at LNF, Nucl. Instrum. Methods Phys. Res., Sect. A 909, 134 (2018).

[25] R. Pompili, C. Chiadroni, C. Cianchi, M. Ferrario, A. Gallo, V. Shpakov, and F. Villa, From SPARC_LAB to EuPRAXIA@ SPARC_LAB, Instruments 3, 45 (2019).

[26] M. Ferrario, EuPRAXIA at SPARC_LAB Conceptual Design Report, Laboratori Nazionali di Frascati Technical Report No. LNF-18/03, LNF-INFN, 2018.

[27] J. Rosenzweig, G. Andonian, M. Ferrario, P. Muggli, O. Williams, V. Yakimenko, and K. Xuan, Plasma wakefields in the quasi-nonlinear regime, AIP Conf. Proc. 1299, 500 (2010).

[28] C. Vaccarezza, D. Alesini, A. Bacci, A. Cianchi, E. Chiadroni, M. Croia, M. Diomede, M. Ferrario, A. Gallo, A. Giribono et al., EUPRAXIA@SPARC_LAB: Beam dynamics studies for the X-band Linac, Nucl. Instrum. Methods Phys. Res., Sect. A 909, 314 (2018).

[29] A. Giribono, A. Bacci, E. Chiadroni, A. Cianchi, M. Croia, M. Ferrario, A. Marocchino, V. Petrillo, R. Pompili, S. Romeo et al., EuPRAXIA@SPARC_LAB: The highbrightness RF photo-injector layout proposal, Nucl. Instrum. Methods Phys. Res., Sect. A 909, 282 (2018).

[30] A. Marocchino, F. Massimo, A. Rossi, E. Chiadroni, and M. Ferrario, Efficient modeling of plasma wakefield acceleration in quasi-non-linear-regimes with the hybrid code ARCHITECT, Nucl. Instrum. Methods Phys. Res., Sect. A 829, 386 (2016).

[31] N. Barov and J.B. Rosenzweig, Propagation of short electron pulses in underdense plasmas, Phys. Rev. E 49, 4407 (1994).

[32] M. Litos, R. Ariniello, C. Doss, K. Hunt-Stone, and J. Cary, Beam emittance preservation using Gaussian density ramps in a beam-driven plasma wakefield accelerator, Phil. Trans. R. Soc. A 377, 20180181 (2019).

[33] R. Pompili, M. P. Anania, E. Chiadroni, A. Cianchi, M. Ferrario, V. Lollo, A. Notargiacomo, L. Picardi, C. Ronsivalle, J. B. Rosenzweig, V. Shpakov, and A. Vannozzi, Compact and tunable focusing device for plasma wakefield acceleration, Rev. Sci. Instrum. 89, 033302 (2018).

[34] W. K. H. Panofsky and W. Baker, A focusing device for the external 350-Mev proton beam of the 184-inch cyclotron at berkeley, Rev. Sci. Instrum. 21, 445 (1950).

[35] M. De Loos and S. Van der Geer, General Particle Tracer: A new 3D code for accelerator and beamline design, in
Proceedings of the 5th European Particle Accelerator Conference (1996).

[36] Y. Fang, J. Vieira, L. Amorim, W. Mori, and P. Muggli, The effect of plasma radius and profile on the development of self-modulation instability of electron bunches, Phys. Plasmas 21, 056703 (2014).

[37] J. Lim, P. Frigola, G. Travish, J. Rosenzweig, S. Anderson, W. Brown, J. Jacob, C. Robbins, and A. Tremaine, Adjustable, short focal length permanent-magnet quadrupole based electron beam final focus system, Phys. Rev. Accel. Beams 8, 072401 (2005).

[38] R. Pompili, M. Anania, E. Chiadroni, A. Cianchi, M. Ferrario, V. Lollo, A. Notargiacomo, L. Picardi, C. Ronsivalle, J. Rosenzweig et al., Compact and tunable focusing device for plasma wakefield acceleration, Rev. Sci. Instrum. 89, 033302 (2018).

[39] E. Boggasch, J. Jacoby, H. Wahl, K.-G. Dietrich, D. Hoffmann, W. Laux, M. Elfers, C. Haas, V. Dubenkov, and A. Golubev, Z-Pinch Plasma Lens Focusing of a Heavy-Ion Beam, Phys. Rev. Lett. 66, 1705 (1991).

[40] E. Boggasch, A. Tauschwitz, H. Wahl, K.-G. Dietrich, D. Hoffmann, W. Laux, M. Stetter, and R. Tkotz, Plasma lens fine focusing of heavy-ion beams, Appl. Phys. Lett. 60, 2475 (1992).

[41] A. Tauschwitz, S. Yu, S. Eylon, R. Bangerter, W. Leemans, C. Peters, J. Rasmussen, L. Reginato, J. Barnard, and W. Sharp, Plasma lens focusing and plasma channel transport for heavy ion fusion, Fusion Eng. Des. 32, 493 (1996).

[42] A. Marocchino et al., Experimental characterization of the effects induced by passive plasma lens on high brightness electron bunches, Appl. Phys. Lett. 111, 184101 (2017).

[43] R. Pompili, M. Anania, M. Bellaveglia, A. Biagioni, S. Bini, F. Bisesto, E. Brentegani, F. Cardelli, G. Castorina, E. Chiadroni et al., Focusing of High-Brightness Electron Beams with Active-Plasma Lenses, Phys. Rev. Lett. 121, 174801 (2018).

[44] C. Lindstrøm, E. Adli, G. Boyle, R. Corsini, A. Dyson, W. Farabolini, S. Hooker, M. Meisel, J. Osterhoff, J.-H. Röckemann et al., Emittance Preservation in an Aberration-Free Active Plasma Lens, Phys. Rev. Lett. 121, 194801 (2018).

[45] A. Zigler, M. Botton, Y. Ferber, G. Johansson, O. Pollak, E. Dekel, F. Filippi, M. Anania, F. Bisesto, R. Pompili et al., Consolidating multiple femtosecond lasers in coupled curved plasma capillaries, Appl. Phys. Lett. 113, 183505 (2018).

[46] A. Curcio, F. Bisesto, G. Costa, A. Biagioni, M. Anania, R. Pompili, M. Ferrario, and M. Petrarca, Modeling and diagnostics for plasma discharge capillaries, Phys. Rev. E 100, 053202 (2019).

[47] N. Bobrova, A. Esaulov, J.-I. Sakai, P. Sasorov, D. Spence, A. Butler, S. Hooker, and S. Bulanov, Simulations of a hydrogen-filled capillary discharge waveguide, Phys. Rev. E 65, 016407 (2001).

[48] J. Van Tilborg, S. Barber, C. Benedetti, C. Schroeder, F. Isono, H.-E. Tsai, C. Geddes, and W. Leemans, Comparative study of active plasma lenses in high-quality electron accelerator transport lines, Phys. Plasmas 25, 056702 (2018). 
[49] R. Bonifacio, C. Pellegrini, and L. Narducci, Collective instabilities and high-gain regime free electron laser, AIP Conf. Proc. 118, 236 (1984).

[50] K.-J. Kim, Brightness, coherence and propagation characteristics of synchrotron radiation, Nucl. Instrum. Methods Phys. Res., Sect. A 246, 71 (1986).

[51] J. Rosenzweig, N. Barov, M. Thompson, and R. Yoder, Energy loss of a high charge bunched electron beam in plasma: Simulations, scaling, and accelerating wakefields, Phys. Rev. Accel. Beams 7, 061302 (2004).

[52] W. Lu, C. Huang, M. Zhou, W. Mori, and T. Katsouleas, Nonlinear Theory for Relativistic Plasma Wakefields in the Blowout Regime, Phys. Rev. Lett. 96, 165002 (2006).

[53] V. Shpakov, M. Anania, M. Bellaveglia, A. Biagioni, F. Bisesto, F. Cardelli, M. Cesarini, E. Chiadroni, A. Cianchi, G. Costa et al., Longitudinal Phase-Space Manipulation with Beam-Driven Plasma Wakefields, Phys. Rev. Lett. 122, 114801 (2019).

[54] J. Su, T. Katsouleas, J. Dawson, and R. Fedele, Plasma lenses for focusing particle beams, Phys. Rev. A 41, 3321 (1990).

[55] G. Hairapetian, P. Davis, C. Clayton, C. Joshi, S. Hartman, C. Pellegrini, and T. Katsouleas, Experimental Demonstration of Dynamic Focusing of a Relativistic Electron Bunch by an Overdense Plasma Lens, Phys. Rev. Lett. 72, 2403 (1994).

[56] H. Nakanishi, Y. Yoshida, T. Ueda, T. Kozawa, H. Shibata, K. Nakajima, T. Kurihara, N. Yugami, Y. Nishida, T. Kobayashi et al., Direct Observation of Plasma-Lens Effect, Phys. Rev. Lett. 66, 1870 (1991).

[57] C. Doss, E. Adli, R. Ariniello, J. Cary, S. Corde, B. Hidding, M. Hogan, K. Hunt-Stone, C. Joshi, K. Marsh et al., Laser-ionized, beam-driven, underdense, passive thin plasma lens, Phys. Rev. Accel. Beams 22, 111001 (2019).

[58] X. Li, A. Chancé, and P. A. P. Nghiem, Preserving emittance by matching out and matching in plasma wakefield acceleration stage, Phys. Rev. Accel. Beams 22, 021304 (2019).
[59] P. Tenenbaum, Collimator wakefield calculations for ilc-trc report, Technical Report No. SLAC-TN-03-038, 2002.

[60] L. Palumbo, V. Vaccaro, and M. Zobov, Wake fields and impedance, Technical Report No. CERN-95-06, CERN, Geneva, 1995.

[61] B. W. Zotter and S. Kheifets, Impedances and Wakes in High Energy Particle Accelerators (World Scientific, Singapore, 1998).

[62] CST Studio Suite, http://www.cst.com.

[63] A. Brynes, B. Spataro, O. Frasciello, A. Marcelli, and G. Castorina, Studies of geometric wakefields and impedances due to collimators, Technical Report No. INFN-1610/LNF, 2016.

[64] S. Agostinelli, J. Allison, K. a. Amako, J. Apostolakis, H. Araujo, P. Arce, M. Asai, D. Axen, S. Banerjee, G. Barrand et al., GEANT4-a simulation toolkit, Nucl. Instrum. Methods Phys. Res., Sect. A 506, 250 (2003).

[65] J. Allison, K. Amako, J. Apostolakis, P. Arce, M. Asai, T. Aso, E. Bagli, A. Bagulya, S. Banerjee, G. Barrand et al., Recent developments in GEANT4, Nucl. Instrum. Methods Phys. Res., Sect. A 835, 186 (2016).

[66] J. R. Votaw and R. J. Nickles, A theoretical description of the beam induced heating of accelerator target foils, Nucl. Instrum. Methods Phys. Res., Sect. A 281, 216 (1989).

[67] R. C. Weast, M. J. Astle, W. H. Beyer et al., CRC Handbook of Chemistry and Physics (CRC Press, Boca Raton, FL, 1988), Vol. 69.

[68] F. Nix and D. MacNair, The thermal expansion of pure metals. II: Molybdenum, palladium, silver, tantalum, tungsten, platinum, and lead, Phys. Rev. 61, 74 (1942).

[69] P. A. Tipler and G. Mosca, Physics for Scientists and Engineers (MacMillan, London, 2007).

[70] D. R. Lide, CRC Handbook of Chemistry and Physics: A Ready-Reference Book of Chemical and Physical Data (CRC Press, Boca Raton, FL, 1995).

[71] K. Floettmann, Adiabatic matching section for plasma accelerated beams, Phys. Rev. Accel. Beams 17, 054402 (2014). 